\title{
The Potential Molecular Mechanism of Autologous Adipose Tissue Grafting in Treating Keloid Patients: a Case-control Study
}

Jiong Zhou ( $\square$ jojozju@zju.edu.cn )

2nd Affiliated Hospital, School of Medicine, Zhejiang University https://orcid.org/0000-0002-95540055

\section{Ji-Yang Shen}

Ningbo Medical Center Lihuili Hospital

\section{Li-En Tao}

2nd Affiliater Hospital, School of Medicine, Zhejiang University

\section{Huan Chen}

The Central Hospital of Lishui City

\section{Research}

Keywords: keloids, wounds, fibroblasts, adipose-derived stem cells

Posted Date: January 19th, 2021

DOl: https://doi.org/10.21203/rs.3.rs-149077/v1

License: (9) This work is licensed under a Creative Commons Attribution 4.0 International License. Read Full License 


\section{Abstract}

Background: Keloids represent the dysregulation of cutaneous wound healing caused by aberrant fibroblast activities. Adipose-derived stem cells have been recognized as a promising treatment for keloids. However, their molecular mechanisms have not been fully elucidated.

Methods: Skin biopsies were obtained from 10 keloid patients and 9 healthy volunteers. Fibroblasts isolated from all samples were divided into 2 groups, one co-cultured with adipose-derived stem cells, the other growing independently. Between each group, we compared the wound healing rate, fibroblasts' survival rate, apoptosis rates, mRNA expressions and protein level of Col-1, Col-3, CTGF, P-4-HB.

Results: In our research, no significant differences between normal fibroblasts and keloid fibroblasts in terms of wound-healing rate, survival rate, or apoptosis rate were found at the baseline. Adipose-derived stem cells strongly suppressed keloid fibroblasts' proliferative and invasive behavior, but negatively regulated keloid fibroblast apoptosis. The further measurement of key components in keloid formation showed that adipose-derived stem cells upregulated Col-3 and CTGF levels in normal fibroblasts but downregulated protein expression of CTGF and P-4-HB in keloid fibroblasts.

Conclusions: Adipose-derived stem cells had the potential to serve as a promising alternative for keloid treatment.

\section{Background}

Keloids refers to dysregulation of cutaneous wound healing, which may develop up to several years after minor injuries (such as trauma, burns, and surgery) even without the patient's awareness. Keloids commonly exceed the wound border, lack natural regression, and are accompanied by itch and pain. Therefore, patients may suffer from substantial physical and psychological distress.

Management for keloids include silicone, corticosteroids, botulinum toxin A, pulsed-dye laser, $\mathrm{CO}_{2}$ laser, 5fluorouracil, bleomycin, surgery, radiation therapy, interferon, interleukin-10, and autologous adiposetissue grafting (AATG)[1]. Today, AATG appears to be a promising alternative because it is minimally invasive and effective[2]. It has been recognized that adipose-derived stem cells (ADSCs) can impact wound healing, soft-tissue restoration, and scar remodeling, due to their angiogenic and anti-apoptotic properties[3]. However, the molecular mechanism of AATG in treating keloid remains unclear.

In the dermis, collagen type I (Col-1, 80\%) and type III (Col-3, 20\%) are the two leading collagen compositions. Fibroblasts (FBs) are the key cells of skin collagen synthesis. The genes responsible for the production of Col-1 and Col-3 are Col1A1 and Col3A1 genes, respectively[4].

Histologically, multiform, thicker collagen fibers are stacked tightly in keloids. Compared to normal skin, Col-3 is over-synthesized, whereas Col-1 levels are reduced[5]. Unshaped extracellular matrix (ECM) surround FBs in the absence of appendages. However, one study demonstrated that Col-1 in keloid 
patients displayed no significant difference compared to a normal volunteer group, whereas Col-3 was significantly higher in patients with keloid compared to the control group[4].

Several signal pathways have been found to be involved in keloid formation. To date, studies on how transforming growth factor (TGF)- $\beta 1 / \mathrm{Smad} 3$ signaling pathways interact with keloid are the most detailed, in which TGF- $\beta 1$ plays an important role in keloid formation. Its effects include chemokine secretion to recruit inflammatory cells and FBs to the wound site[6], stimulation of collagen synthesis, and suppression of collagen degradation[7]. TGF- $\beta 1$ also induces autocrine of connective tissue growth factor (CTGF) in keloid FBs[8].

Prolyl 4-hydroxylase B (P-4-HB) is necessary for the stabilization of the collagen triple helix structure, enhancing the effect of collagen crossing and scarring[9]. In recent years, it has been discovered that ADSCs have the ability to reverse keloid formation[10,11,12]. However, its mechanisms have not been fully elucidated. Therefore, with the aim of identifying efficient targets in keloid treatment, our research was conducted to explicitly describe the pathways associated with ADSC alleviation of keloids.

\section{Methods}

\section{Sample collection, primary fibroblasts culture, and reagents}

Skin samples were obtained from 10 keloid patients and 9 healthy volunteers ( 9 males and 10 females, 18-55 years old) at cosmetic surgical operations (face or chest) after signing informed consents.

Samples were placed in $0.5 \%$ dispase (Invitrogen, Carlsbad, CA) at $4{ }^{\circ} \mathrm{C}$, and the dermis was separated from the epidermis after overnight incubation with $0.1 \%$ collagenase (Invitrogen). The released FBs were cultured in DMEM containing 10\% fetal bovine serum. FBs between the second and the sixth passages were used in subsequent experiments. FBs from healthy volunteers were sorted into Groups F1, F2, and F3. FBs from keloid tissues were grouped into F4, F5, and F6[13].

\section{Adipose-derived stem cells isolation and identification}

All human adipose tissues were from three volunteers ( 1 male and 2 females) without systemic diseases, aged 20 to 25 years old. They underwent liposuction surgery after providing their informed consent and with the due approval of the Research Ethical Committee of the 2nd Affiliated Hospital, School of Medicine, Zhejiang University.

Fifty $\mathrm{ml}$ human lipoaspirate was washed three times in phosphate-buffered saline (PBS; Hyclone, USA) and finely minced, before being digested in $0.1 \%$ collagenase I (Sigma, USA) at $37.0^{\circ} \mathrm{C}$ with continuous vibration for approximately $1 \mathrm{~h}$. Digestion was terminated by adding Dulbecco's Modified Eagle Medium/F12 (DMEM; Hyclone, USA) containing 10\% Mesenclaymal stem cell Bovine Serum (MFBS; BI, USA). The tissue was centrifuged at 1,200 revolutions per minute for $5 \mathrm{~min}$, at the temperature of $25^{\circ} \mathrm{C}$. Then, the supernatant was discarded, and the cell pellet was washed in PBS before being centrifuged at 1,000 revolutions per minute for $5 \mathrm{~min}$, at the temperature of $25^{\circ} \mathrm{C}$. Cells were resuspended in common 
culture media (DMEM containing 10\% MFBS) and counted; then, they were inoculated at a density of $1 \times 10^{9}$ cells per liter. The first culture medium change was conducted $24 \mathrm{~h}$ after inoculating and was routinely repeated every $72 \mathrm{~h}$. When cells reached $80-90 \%$ confluence, the subculture was operated using $0.25 \%$ trypsin with $0.03 \%$ EDTA (Gibco, USA). Human ADSCs (hADSCs) between the third and the fourth passages were used in the subsequent experiments.

hADSCs (passage 2) were harvested and washed in PBS three times. Cell suspension was incubated with FITC-conjugated antibodies against CD45 (11-0459-42, eBioscience), CD34 (22-0349-42, eBioscience), PEconjugated antibodies against CD105 (12-1057-42, eBioscience), PE Cyanine5-conjugated antibodies against HLA-DR (11-0459-42, eBioscience) at $37.0^{\circ} \mathrm{C}$ for $15 \mathrm{~min}$ in the dark, followed by washing and resuspension in PBS. Then, it was finally detected by flow cytometer (BD Biosciences, USA).

\section{Immunohistochemistry analysis}

The paraffin-embedded samples were sliced into 4- $\mu \mathrm{m}$ sections and incubated with primary antibodies: Anti-Col-1 (ab34710, Abcam. Dilution 1:100), Anti-Col-3 (ab7778, Abcam. Dilution 1:100), Anti-CTGF (ab6992, Abcam. Dilution 1:100), and Anti-P4HA2 (ab211527, Abcam. Dilution 1:100) after dewaxing, hydration, antigen retrieval, and blocking. The expression was detected using the immunoperoxidase technique (DAB kit, China).

\section{RNA Extraction}

RNA extraction, reverse transcription, and qRT-PCR were conducted as described in [13].

\section{Co-culture of fibroblast and ADSC, CCK8 assay for cell growth curve}

Normal FBs (F1, F2, and F3) and keloid FBs (F4, F5, and F6) were co-cultured with ADSCs (grouped into $A 1, A 2$, and $A 3$ ) in a 24-well plate (Table 1). The FB suspension was seeded in a 24-well plate $\left(5 \times 10^{4} /\right.$ well). Then, $5 \times 10^{4} /$ well ADSCs were plated onto the chamber $(0.4 \mu \mathrm{M})$. The co-culture lasted for $48 \mathrm{~h}$ when $10 \%$ CCK8 solution was added to each well, and the plates were incubated for $1 \mathrm{~h}$. Finally, the absorbance was measured at $450 \mathrm{~nm}$ using a microplate reader. 
Table 1

Co-cultural scheme in a 24-well plate.

\begin{tabular}{|c|c|c|c|c|c|c|}
\hline & \multicolumn{3}{|l|}{ nFBs } & \multicolumn{3}{|l|}{ kFBs } \\
\hline & F1 & F2 & F3 & F4 & F5 & F6 \\
\hline NC & $\mathrm{F} 1+\mathrm{NC}$ & $\mathrm{F} 2+\mathrm{NC}$ & $\mathrm{F} 3+\mathrm{NC}$ & $\mathrm{F} 4+\mathrm{NC}$ & $\mathrm{F} 5+\mathrm{NC}$ & $\mathrm{F} 6+\mathrm{NC}$ \\
\hline $\mathrm{A} 1$ & $\mathrm{~F} 1+\mathrm{A} 1$ & $\mathrm{~F} 2+\mathrm{A} 1$ & $\mathrm{~F} 3+\mathrm{A} 1$ & $\mathrm{~F} 4+\mathrm{A} 1$ & $\mathrm{~F} 5+\mathrm{A} 1$ & $\mathrm{~F} 6+\mathrm{A} 1$ \\
\hline $\mathrm{A} 2$ & $\mathrm{~F} 1+\mathrm{A} 2$ & $\mathrm{~F} 2+\mathrm{A} 2$ & $\mathrm{~F} 3+\mathrm{A} 2$ & $\mathrm{~F} 4+\mathrm{A} 2$ & $\mathrm{~F} 5+\mathrm{A} 2$ & $\mathrm{~F} 6+\mathrm{A} 2$ \\
\hline A3 & $\mathrm{F} 1+\mathrm{A} 3$ & $\mathrm{~F} 2+\mathrm{A} 3$ & $\mathrm{~F} 3+\mathrm{A} 3$ & $\mathrm{~F} 4+\mathrm{A} 3$ & $\mathrm{~F} 5+\mathrm{A} 3$ & $\mathrm{~F} 6+\mathrm{A} 3$ \\
\hline
\end{tabular}

\section{Cell apoptosis detection}

After $48 \mathrm{~h}$ of co-culture with ADSCs, FBs were collected and resuspended in $1 \times$ binding buffer at a concentration of $1.0 \times 10^{6}$ cells $/ \mathrm{mL}$, and $100 \mu \mathrm{L}$ of sample was mixed with $5 \mu \mathrm{L}$ of FITC Annexin $V$ and $5 \mu \mathrm{L}$ of propidium iodide (PI). The sample was mixed gently and shielded from light at room temperature for $5 \mathrm{~min}$. Then, $400 \mu \mathrm{L}$ of $1 \times$ binding buffer was added. The sample was detected and analyzed within $1 \mathrm{~h}$ by flow cytometry.

\section{In vitro wound healing assay}

The wound-healing assay was conducted as described in[14].

\section{Western blot analysis}

Western blot was conducted as described in[14]. Antibodies against Col-1, Col-3, CTGF, and P-4-HB were purchased from Abcam, and antibodies against $\beta$-actin were purchased from Multi Sciences.

\section{Statistical analysis}

All of the experiments were performed in triplicate at minimum. Data were presented as the mean \pm standard deviations from more than two independent experiments.

The Kolmogorov-Smirnov test using SPSS 16.0 (SPSS, Inc) software was used to determine whether or not data fitted normal distribution. GraphPad Prism 7 (GraphPad Software, USA) software was used to evaluate statistically significant differences by $t$-test and to generate graphs. Results were considered statistically significant when the probability was less than $5 \%(p<0.05)$.

\section{Results}




\section{Fibroblasts and adipose-derived stem cells can reverse keloid formation}

A 16-year-old girl was accepted for debridement several times due to a severe traffic accident, which led to her keloids limiting her movement. After autologous adipose tissue grafting, the scar became softer, and her movement obviously improved (Supplementary Fig. 1). These results suggest that incorporation of FBs and ADSCs can positively impact pathological scar restoration. The associated molecular mechanism is worth investigating.

\section{Characterization and identification of hADSC}

ADSCs were adherent to plastic and demonstrated FB-like morphology after two passages, as illustrated in Supplementary Fig. 2. Immunophenotype was assessed, and results revealed that the cells were positive for MSC marker CD105 and negative for HLA-DR, CD45, CD34, according to the international criteria for defining multipotent MSC[15] (Supplementary Fig. 3).

\section{hADSCs enhance the function of normal fibroblasts more than keloid fibroblasts to repair wounds}

Wound-healing rate was compared using scratch tests (Fig. 1). In co-cultural groups with $A 2$ and A3, the wound-healing rates of normal FBs were significantly higher than that of keloid FBs. Groups co-cultured with $\mathrm{A} 1$ demonstrated a slight trend toward significance. In the normal control (NC) group, we observed no significance. Healing rates of normal FBs co-cultured with A1, A2, and A3 apparently surpassed those in the NC group. Results revealed conclusively that hADSCs enhanced the function of normal FBs more impressively compared to keloid FBs to repair wounds.

\section{hADSCs improve the survival rate of normal fibroblasts more effectively than keloid fibroblasts.}

CCK8 tests were used to examine the cellular survival rate of normal and keloid FBs in the NC group or when co-cultured with ADSCs (Fig. 2). Comparison of cellular survival rates of normal keloid FBs in the NC group revealed no significant differences. However, in all three co-cultured groups, cellular survival rates were all obviously higher for normal FBs $(p<0.01)$. Compared to keloid FBs in the NC group, the survival rates of keloid FBs co-cultured with A1, A2, and A3 were all lower $(p<0.01)$. Such a result demonstrated that hADSCs could effectively repress the survival rate of keloid FBs. We assumed that hADSCs may improve keloid recovery by inducing apoptosis in keloid FBs rather than activating normal FBs.

\section{A balance may exist between hADSCs' regulation of keloid fibroblasts' survival and keloid apoptosis}

In terms of apoptosis rate, the difference between normal FBs and keloid FBs was nonsignificant in all of the four groups (Fig. 3). However, apoptosis rates of keloid FBs in three co-cultured groups were lower than those in the NC group. Based on the results of the CCK8 test, which suggested that hADSCs could decrease keloid FB survival rates, there appeared to be a balance between hADSCs' regulation of keloid FB survival and keloid FB apoptosis. 
Col-3 and P-4-HB were over-synthesized in keloid.

Immunohistochemistry demonstrated that levels of Col-3 and P-4-HB were higher in keloid than in normal tissue (Fig. 4). Levels of Col-1 and CTGF were basically the same.

mRNA expressions of Col-1, Col-3, CTGF, and P-4-HB in both normal fibroblasts and keloid fibroblasts do not demonstrate much significance.

The mRNA level of Col-1, Col-3, CTGF, and P-4-HB in normal and keloid FBs did not display great significance at the baseline and in co-cultural groups (Fig. 5). In co-cultured groups, compared to normal FBs, keloid FBs appeared to be downregulated; however, only the A2 group demonstrated any significant difference.

When co-cultured with A3, P-4-HB expression was suppressed in keloid FBs, compared to keloid FBs in the NC group. This may infer that one of the pathways by which hADSCs treat keloids stimulates Col-1 expression in normal FBs. Another probable mechanism is the suppression of P-4-HB expression in keloid FBs.

The protein levels of Col-3, CTGF, and P-4-HB are lower in keloid fibroblasts co-cultured with hADSCs than normal fibroblasts under the same condition.

Western blot verified protein levels of Col-1, Col-3, CTGF, and P-4-HB in each group (Fig. 6). Parallel to the qRT-PCR results, the protein levels of Col-3 and P-4-HB were lower in keloid FBs co-cultured with hADSCs than in normal FBs under the same condition. Furthermore, Col-1 protein levels were not altered. Results strongly demonstrated that hADSCs can help suppress keloids by inhibiting Col-3 and P-4HB production in keloid FBs. Intriguingly, in co-cultured groups, the CTGF protein level was also lower in keloid FBs than in normal FBs, which was not indicated by qRT-PCR.

With ADSCs, the expression of Col-1, Col-3, CTGF, and P-4-HB protein in normal FBs were all upregulated, whereas only Col-3 and CTGF exhibited significant differences. However, protein expression all decreased in keloid FBs, whereas CTGF and P4HB displayed significant differences.

\section{ADSCs restrained migration of keloid dermal fibroblasts, but promoted migration of normal dermal fibroblasts.}

The effects of ADSC co-culture on migration of normal and keloid dermal FBs were investigated using scratch assays. In these assays, the artificial scratch was closed by proliferation and migration of the cultured cells. As shown in Fig. 7, at the baseline, keloid FBs displayed better migration capability compared to normal FBs.

ADSC promoted closure of scratches in normal FBs by $43.3 \%( \pm 15.3)$; however, the cells restrained the migration of keloid FBs by $28.1 \%( \pm 9.5)$ as compared with controls after $24 \mathrm{~h}$. However, no significant differences in closure rate were detected. 


\section{Discussion}

Keloids are human-specific pathological healing of skin lesions. The incidence of keloids in the Chinese population ranges between $4.6 \%$ and $16 \%[16]$. Keloid FBs are refractory to apoptosis. FBs located in the keloid margin are more highly proliferative than FBs in the center[5]. Therefore, keloid borders develop beyond the original traumas. Keloids in exposed sites can be disfiguring and accompanied by itching and pain. Abnormal proliferation and hardening of collagens limit muscular function. Keloid patients suffer from physiological, mental, and social stresses.

Autologous fat transplantation has proven effective for pathologic scars both experimentally and clinically[17]. However, the related molecular mechanisms are yet to be illustrated in detail. Further elucidation of the involved molecular mechanisms would confirm its effectiveness and even help develop potential therapies.

Wang's team[18] revealed that ADSC-CM attenuated the bioactivities of keloid FBs by downregulating extracellular matrix-related gene expression, including plasminogen activator inhibitor-1, tissue inhibitor of metalloproteinase-1, and Col-1, and by inhibiting the expression of cell proliferation proteins. However, their research did not include comparisons with normal FBs.

In our study, we studied both normal and keloid FBs with hADSCs, and duplicated these experiments with three different strains of hADSCs, normal FBs, and keloid FBs. Analyses were conducted independently for each co-cultured group and for the NC group. Our results uncovered no significant differences between normal FBs and keloid FBs in terms of wound-healing rate, survival rate, or apoptosis rate at the baseline. Interestingly, with ADSC, wound-healing rate and survival rate of normal FBs were promoted, while keloid FBs reduced evidently although the characterization of keloid FBs is high capacity of proliferation and aggressive properties[19]. Both normal FBs and keloid FBs exhibited restrained apoptosis rates, with keloid FBs exhibiting greater restraint of apoptosis rates. It can be inferred that ADSC restrained multiple bioactivities in keloid FBs and intensified normal FB functions. This effect may explain why ADSC can promote wound healing in normal people but inhibit keloid formation in patients in a process that could be termed "perfect wound healing."

The scratch tests also proved hADSCs' ability to promote wound healing primarily by enhancing normal FB functions, as opposed to keloid FB functions. We found that the cellular survival rates of normal and keloid FBs displayed no difference in nonco-cultured groups. However, co-culture with hADSCs exhibited a markedly suppressing effect on keloid FB survival, consistent with scratch test findings. The combination of the results above suggested that hADSCs strongly suppressed keloid FB's proliferative ability and invasive behavior.

However, hADSCs also regulated keloid FB's apoptosis negatively, which could counteract the suppressed survival rate. Overall, hADSC interactions with normal and keloid FB cellular functions are beneficial to wound recovery and keloid improvement, despite the inhibition of keloid FB apoptosis. 
Based on discoveries relating to cellular activities, we further tested four fibrous markers, namely, Col-1, Col-3, CTGF, and P-4-HB, in terms of their effects on mRNA and protein levels. Collagen is the dominant protein of ECM[20] and is essential for cellular physiological function, including motility, metabolism, proliferation, differentiation, orientation, and survival. Fibrosis is a pathological process in the ECM leading to impairment of organ function[21]. In traumatic skin, the synthesis of Col-3 even exceeds that of Col-1 in contrast to normal skin[22, 23]. The dry weight ratio of Col-3 to Col-1 increases from approximately 1:4 in unlesioned dermis to $1: 1$ in injured dermis[5]. CTGF is a member of the CCN protein family. It has been reported that CTGF can regulate multiple FB behaviors that can contribute to the development of fibrosis, including proliferation, differentiation, migration, adhesion, and matrix production. CTGF is highly expressed in various fibrotic conditions regulated by various profibrotic molecules, such as TGF- $\beta_{1}$ [24], MAPK pathway[25], angiotensin II, and endothelin-1[26]. During fibrosis, FBs express excessive P-4-HB to stabilize collagen triple helix structure[27]. Deactivation of P-4-HB reduces synthesis and secretion of pro-collagen Type $\triangle$ and $\triangle[28]$. Results from qRT-PCR indicated that one of the pathways by which hADSCs treated keloids was via stimulation of Col- 1 expression in normal FBs. Another probable mechanism was suppression of P-4-HB expression in keloid FBs. Western blot results strongly demonstrated that hADSCs could suppress keloids by inhibiting Col-3 and P-4-HB protein productions in keloid FBs. Intriguingly, in co-cultured groups, CTGF protein levels were also lower in keloid FBs than in normal FBs, which was not indicated by qRT-PCR. Whether the alterations of CTGF synthesis are caused by regulation of its upstream molecules is worth further investigation. Results comprehensively demonstrated that hADSCs contribute to keloid recovery by restricting several profibrotic molecules.

\section{Conclusions}

Our current study proved hADSCs' effectiveness in reversing keloid formation in accordance with the clinical outcome of autologous adipose tissue grafting. We found that hADSCs regulated several cellular activities of normal or keloid FBs separately, from which we conjectured that keloid FBs were more of a target of hADSCs than normal FBs. On the molecular level, our study involved two types of fibrosis-related genes: the ECM-related genes, including Col-1 and Col-3 genes and fibrosis markers, including CTGF and P-4-HB. Results revealed that ADSC upregulated protein expression of Col-3 and CTGF in normal FBs but downregulated protein expression of CTGF and P-4-HB in keloid FBs. If new methods were discovered for specific inhibition against ADSCs' suppressing effect on the apoptosis rate of keloid FBs, it would help further shorten the therapy period. Consequently, ADSC not only promoted the bioactivities of normal FBs but also attenuated those of keloid FBs. As such, ADSCs can be promising alternatives in both rejuvenation and keloid treatment.

\section{Abbreviations}

AATG: autologous adipose-tissue grafting:

ADSCs : adipose-derived stem cells 
Col-1: collagen type I

Col-3: collagen type III

FBs: Fibroblasts

ECM: extracellular matrix

TGF: transforming growth factor

CTGF: connective tissue growth factor

P-4-HB: Prolyl 4-hydroxylase B:

NC: normal control

\section{Declarations}

\section{Ethics approval and consent to participate}

The study was approved by the ethics committee of the 2nd Affiliated Hospital, School of Medicine, Zhejiang University. Skin samples were obtained from 10 keloid patients and 9 healthy volunteers at cosmetic surgical operations after signing informed consents.

\section{Consent for publication}

Not applicable

\section{Competing interests}

The authors declare that they have no competing interests.

\section{Availability of data and materials}

The datasets used and/or analysed during the current study are available from the corresponding author on reasonable request.

\section{Funding}

This research was supported by the CMA-L'OREAL China Skin/Hair Grant (grant number: S2018-012).

\section{Authors' contributions}

Jiong Zhou: conceptualization, resources, funding acquisition, methodology, data curation.

Ji-Yang Shen: data curation. software, formal analysis, writing - original draft, writing - review \& editing. 
Li-En Tao: investigation, validation, writing - review \& editing.

Huan Chen: investigation, validation, writing - review \& editing.

\section{References}

1. Huang C, Liu L, You Z, Du Y, Ogawa R. Managing keloid scars: From radiation therapy to actual and potential drug deliveries. Int Wound J. 2019;16;(3): 852-59.

2. Riyat $H$, Touil LL, Briggs $M$, Shokrollahi K. Autologous fat grafting for scars, healing and pain: a review. Scars Burn Heal. 2017;3;(2059-5131 (Electronic)).

3. Lee G, Hunter-Smith DJ, Rozen WM. Autologous fat grafting in keloids and hypertrophic scars: a review. Scars Burn Heal. 2017;3: 2059513117700157.

4. da Silva IR, Tiveron L, da Silva MV, Peixoto AB, Carneiro CAX, Dos Reis MA, Furtado PC, Rodrigues BR, Rodrigues V, Jr., Rodrigues DBR. In Situ Cytokine Expression and Morphometric Evaluation of Total Collagen and Collagens Type I and Type III in Keloid Scars. Mediators Inflamm. 2017;2017: 6573802.

5. Rippa AL, Kalabusheva EP, Vorotelyak EA. Regeneration of Dermis: Scarring and Cells Involved. 2019;8;(6): 607.

6. Bullard KM, Longaker MT, Lorenz HP. Fetal wound healing: current biology. World J Surg. 2003;27;(1): 54-61.

7. Szulgit G, Rudolph R, Wandel A, Tenenhaus M, Panos R, Gardner H. Alterations in fibroblast alpha1beta 1 integrin collagen receptor expression in keloids and hypertrophic scars. J Invest Dermatol. 2002;118;(3): 409-15.

8. Luo L, Li J, Liu H, Jian X, Zou Q, Zhao Q, Le Q, Chen H, Gao X, He C. Adiponectin Is Involved in Connective Tissue Growth Factor-Induced Proliferation, Migration and Overproduction of the Extracellular Matrix in Keloid Fibroblasts. Int J Mol Sci. 2017;18;(5): 1044.

9. Heber-Katz E. Oxygen, Metabolism, and Regeneration: Lessons from Mice. Trends Mol Med. 2017;23; (11): 1024-36.

10. Kalinina N, Kharlampieva D, Loguinova M, Butenko I, Pobeguts O, Efımenko A, Ageeva L, Sharonov G, Ischenko D, Alekseev D, Grigorieva O, Sysoeva V, Rubina K, Lazarev V, Govorun V. Characterization of secretomes provides evidence for adipose-derived mesenchymal stromal cells subtypes. Stem Cell Res Ther. 2015;6: 221.

11. Haubner F, Muschter D, Pohl F, Schreml S, Prantl L, Gassner HG. A Co-Culture Model of Fibroblasts and Adipose Tissue-Derived Stem Cells Reveals New Insights into Impaired Wound Healing After Radiotherapy. Int J Mol Sci. 2015;16;(11): 25947-58.

12. Li Y, Zhang W, Gao J, Liu J, Wang H, Li J, Yang X, He T, Guan H, Zheng Z, Han S, Dong M, Han J, Shi J, Hu D. Adipose tissue-derived stem cells suppress hypertrophic scar fibrosis via the p38/MAPK signaling pathway. Stem Cell Res Ther. 2016;7;(1): 102. 
13. Man XY, Li W, Chen JQ, Zhou J, Landeck L, Zhang KH, Mu Z, Li CM, Cai SQ, Zheng M. Impaired nuclear translocation of glucocorticoid receptors: novel findings from psoriatic epidermal keratinocytes. Cell Mol Life Sci. 2013;70;(12): 2205-20.

14. Cao HL, Zhou J, Chen XB, Landeck L, Yang JQ, Chen JQ, Li W, Cai SQ, Zheng M, Man XY. Inhibition of the hedgehog pathway leads to antifibrotic effects in dermal fibrosis. Discov Med. 2016;22;(122): 311-18.

15. Dominici M, Le Blanc K, Mueller I, Slaper-Cortenbach I, Marini F, Krause D, Deans R, Keating A, Prockop D, Horwitz E. Minimal criteria for defining multipotent mesenchymal stromal cells. The International Society for Cellular Therapy position statement. Cytotherapy. 2006;8;(4): 315-7.

16. Lu WS, Zheng XD, Yao XH, Zhang LF. Clinical and epidemiological analysis of keloids in Chinese patients. Arch Dermatol Res. 2015;307;(2): 109-14.

17. Plikus MV, Guerrero-Juarez CF, Ito M, Li YR, Dedhia PH, Zheng Y, Shao M, Gay DL, Ramos R, Hsi TC, Oh JW, Wang X, Ramirez A, Konopelski SE, Elzein A, Wang A, Supapannachart RJ, Lee HL, Lim CH, Nace A, Guo A, Treffeisen E, Andl T, Ramirez RN, Murad R, Offermanns S, Metzger D, Chambon P, Widgerow AD, Tuan TL, Mortazavi A, Gupta RK, Hamilton BA, Millar SE, Seale P, Pear WS, Lazar MA, Cotsarelis G. Regeneration of fat cells from myofibroblasts during wound healing. Science. 2017;355; (6326): 748-52.

18. Wang X, Ma Y, Gao Z, Yang J. Human adipose-derived stem cells inhibit bioactivity of keloid fibroblasts. Stem Cell Res Ther. 2018;9;(1): 40.

19. Le AD, Zhang Q, Wu Y, Messadi DV, Akhondzadeh A, Nguyen AL, Aghaloo TL, Kelly AP, Bertolami CN. Elevated vascular endothelial growth factor in keloids: relevance to tissue fibrosis. Cells Tissues Organs. 2004;176;(1-3): 87-94.

20. Vasta JD, Raines RT. Collagen Prolyl 4-Hydroxylase as a Therapeutic Target. J Med Chem. 2018;61; (23): 10403-11.

21. Herrera J, Henke CA, Bitterman PB. Extracellular matrix as a driver of progressive fibrosis. J Clin Invest. 2018;128;(1): 45-53.

22. Woodley DT. Distinct Fibroblasts in the Papillary and Reticular Dermis: Implications for Wound Healing. Dermatol Clin. 2017;35;(1): 95-100.

23. Xue M, Jackson CJ. Extracellular Matrix Reorganization During Wound Healing and Its Impact on Abnormal Scarring. Adv Wound Care (New Rochelle). 2015;4;(3): 119-36.

24. Zhu Y, Yu F, Tan Y, Hong Y, Meng T, Liu Y, Dai S, Qiu G, Yuan H, Hu F. Reversing activity of cancer associated fibroblast for staged glycolipid micelles against internal breast tumor cells. Theranostics. 2019;9;(23): 6764-79.

25. Fabregat I, Caballero-Díaz D. Transforming Growth Factor- $\beta$-Induced Cell Plasticity in Liver Fibrosis and Hepatocarcinogenesis. Frontiers in oncology. 2018;8: 357.

26. Sakai N, Nakamura M, Lipson KE, Miyake T, Kamikawa Y, Sagara A, Shinozaki Y, Kitajima S, Toyama T, Hara A, Iwata Y, Shimizu M, Furuichi K, Kaneko S, Tager AM, Wada T. Inhibition of CTGF 
ameliorates peritoneal fibrosis through suppression of fibroblast and myofibroblast accumulation and angiogenesis. Sci Rep. 2017;7;(1): 5392.

27. Pilling D, Gomer RH. The Development of Serum Amyloid P as a Possible Therapeutic. Frontiers in immunology. 2018;9: 2328.

28. Shi R, Hu W, Zhang Y, Gao S, Smith AH, Ye J, Cai L, Graham LM, Li C. Ascorbate inducible N259 glycans on prolyl 4-hydroxylase subunit a1 promote hydroxylation and secretion of type I collagen. Cellular and molecular life sciences : CMLS. 2019;76;(17): 3449-64.

\section{Figures}



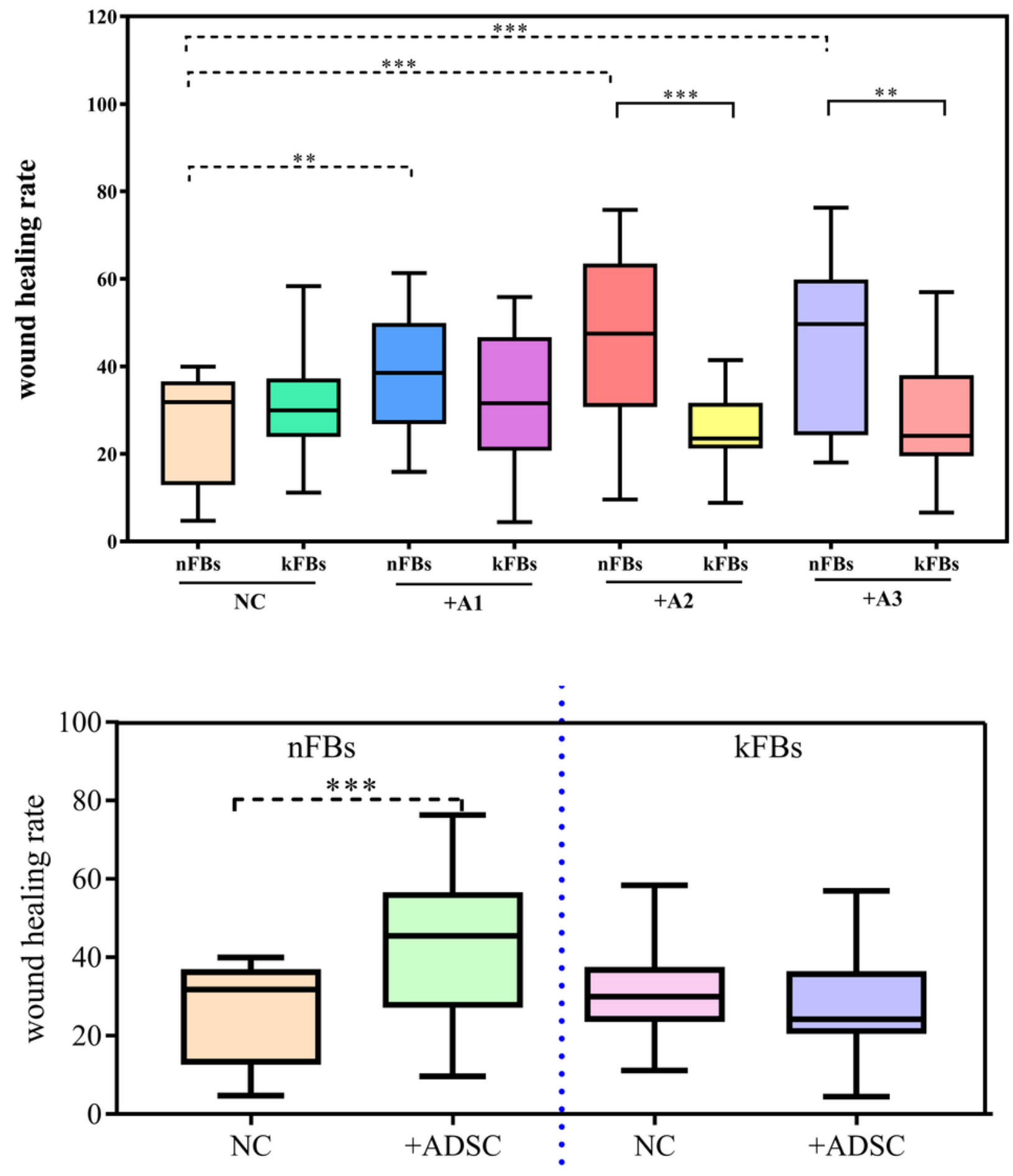

Figure 1

Comparison of wound healing rates. In co-cultural groups with $\mathrm{A} 2$ and $\mathrm{A} 3$, the wound-healing rates of $\mathrm{nFBs}$ were significantly higher than that of kFBs. The wound-healing rates of three strains of $\mathrm{nFBs}$ in cocultured groups were higher than those of nFBs in the NC group. ${ }^{\star} p<0.05 ;{ }^{*} p<0.005$; ${ }^{\star \star \star} p<0.001$. NC: normal control. nFBs: normal fibroblasts; kFBs: keloid fibroblasts. A, ADSC: adipose-derived stem cells. 


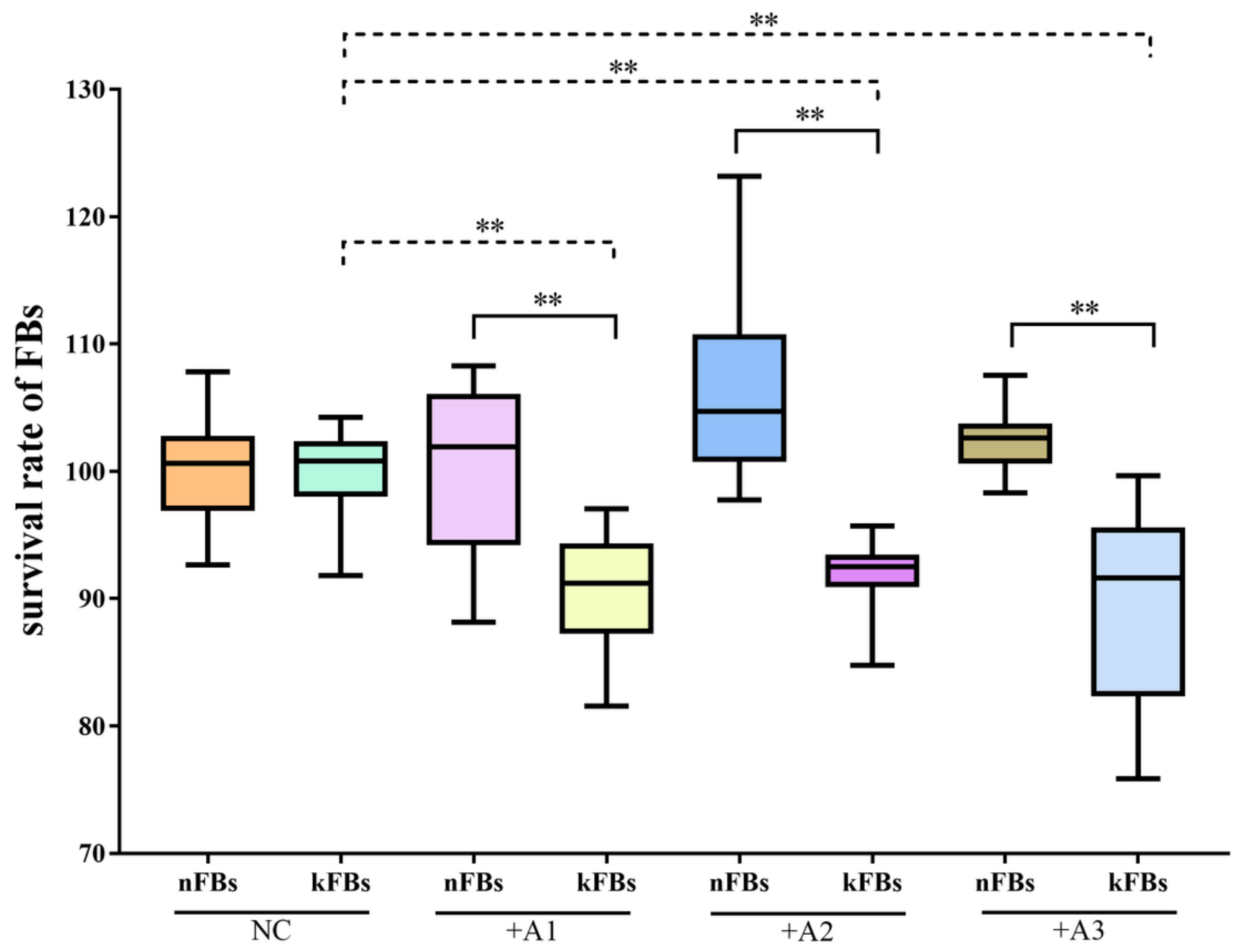

Figure 2

Comparison of fibroblast survival rates. There was no obvious difference between survival rates of normal and keloid FBs in the normal control group. However, the survival rate of nFBs was obviously higher than kFBs co-cultured with ADSC. The survival rates of three strains of kFBs were lower than that of kFBs in the normal control group. ${ }^{*} \mathrm{p}<0.005$. NC: normal control. A: adipose-derived stem cells. nFBs: normal fibroblasts; kFBs: keloid fibroblasts. 


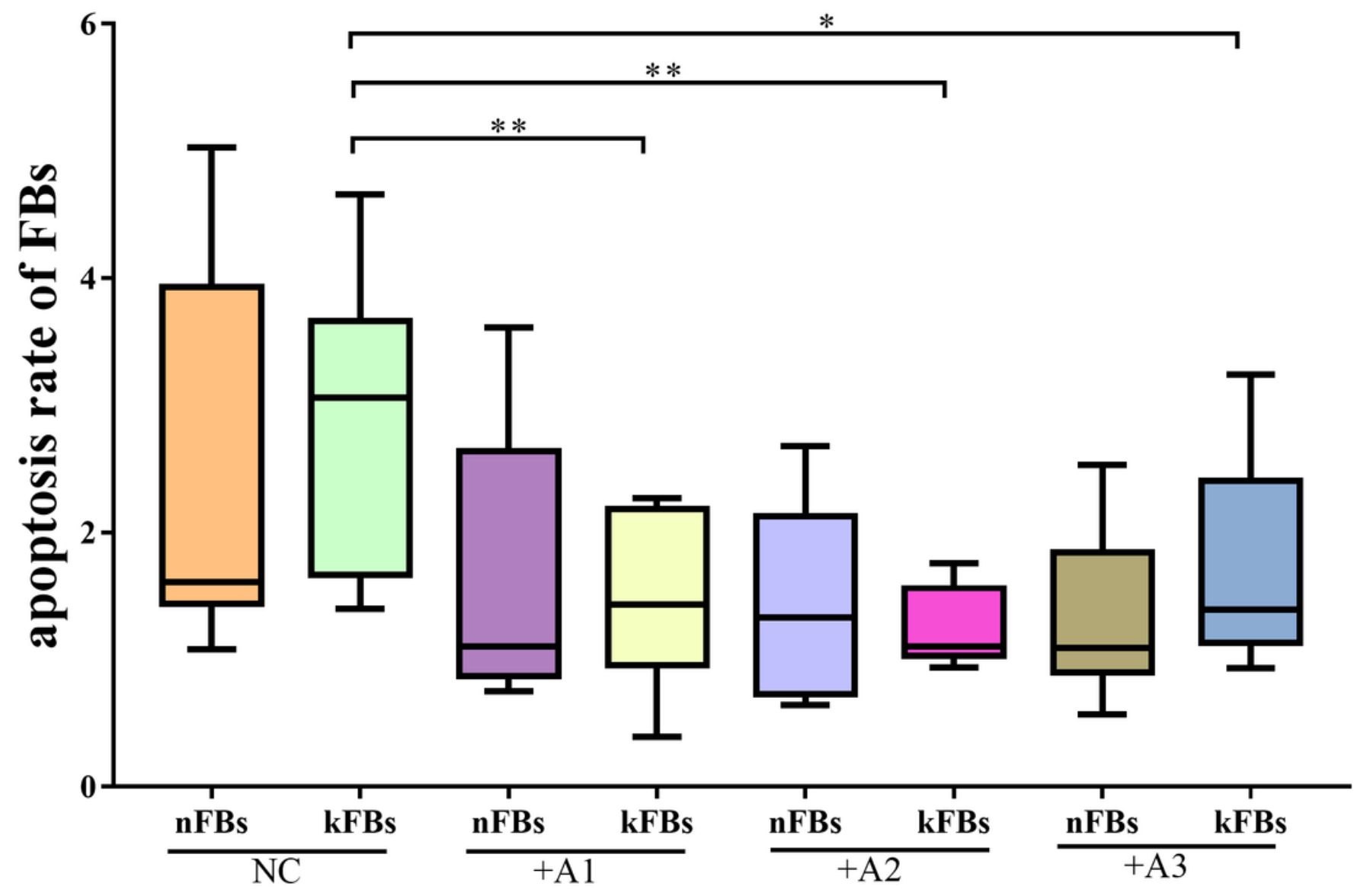

Figure 3

Comparison of cellular apoptosis rates. Differences in cellular apoptosis rate between nFBs and kFBs were nonsignificant in all of the four groups, namely, normal control, A1, A2, and A3. However, apoptosis rates for $\mathrm{kFBs}$ in the three co-cultured groups were lower than that in the normal control group. ${ }^{*} \mathrm{p}<0.05$; ${ }^{*} \mathrm{p}<0.005$. NC: normal control. A: adipose-derived stem cells. nFBs: normal fibroblasts; kFBs: keloid fibroblasts. 


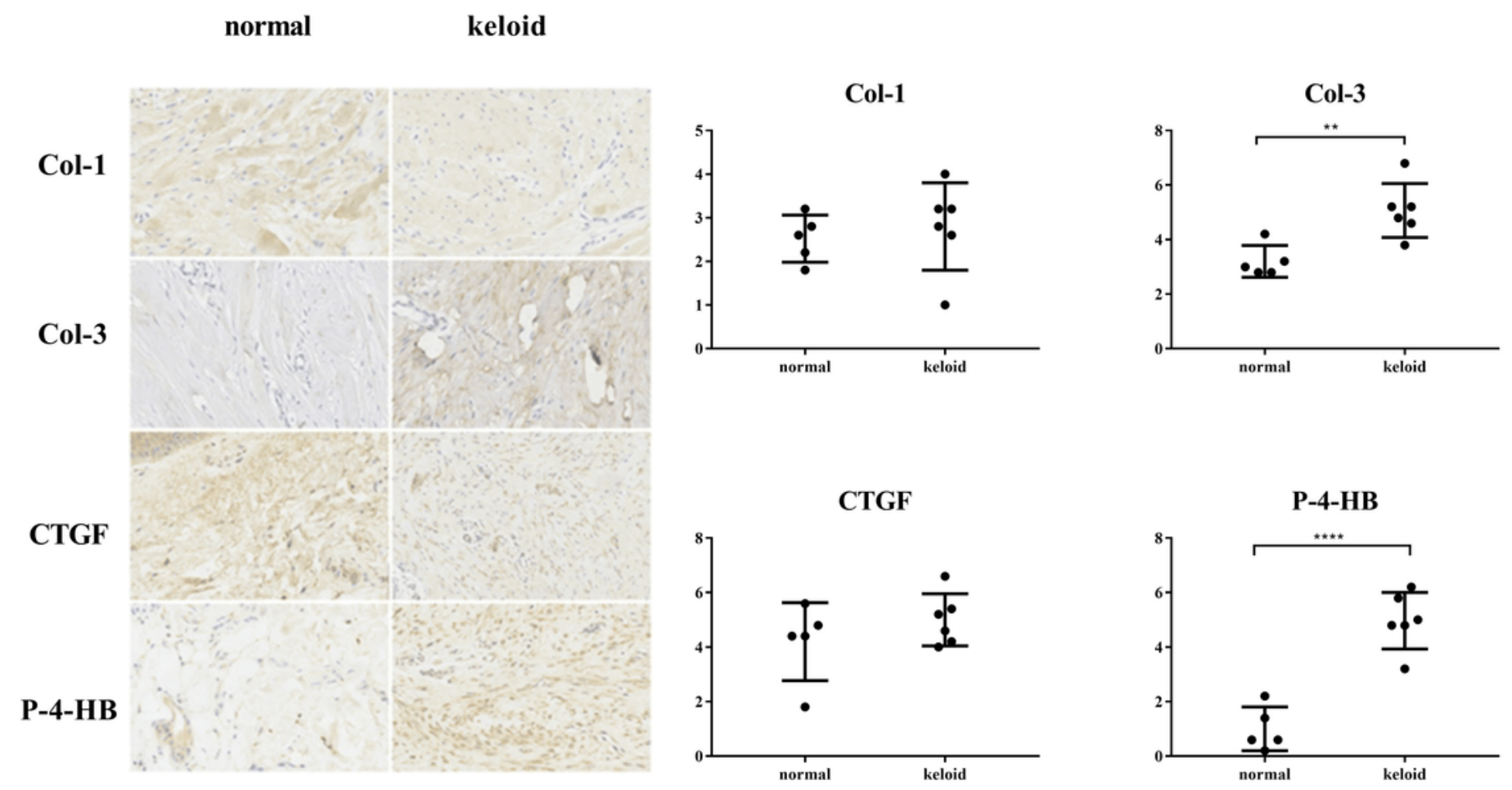

Figure 4

The result of immunohistochemistry. The levels of Col-3 and P-4-HB were higher in keloid tissue than in normal tissue. The levels of Col-1 and CTGF were basically the same. ${ }^{* \star} p<0.005$; ${ }^{\star \star \star \star} p<0.0001$. Col: collagen. CTGF: connective tissue growth factor. P-4-HB: Prolyl 4-hydroxylase B. 
Col-1

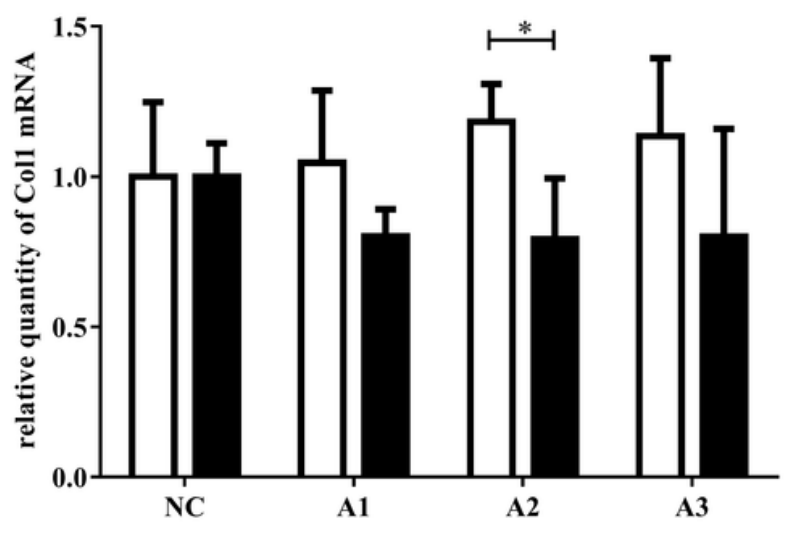

CTGF

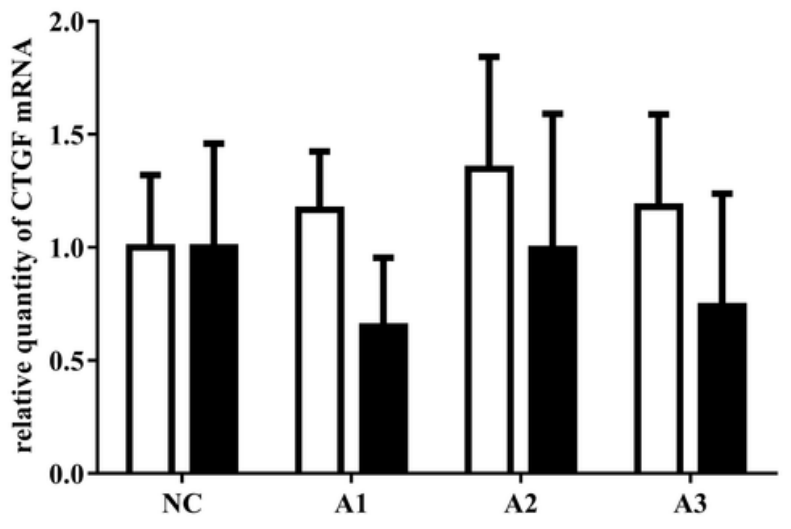

Col-3

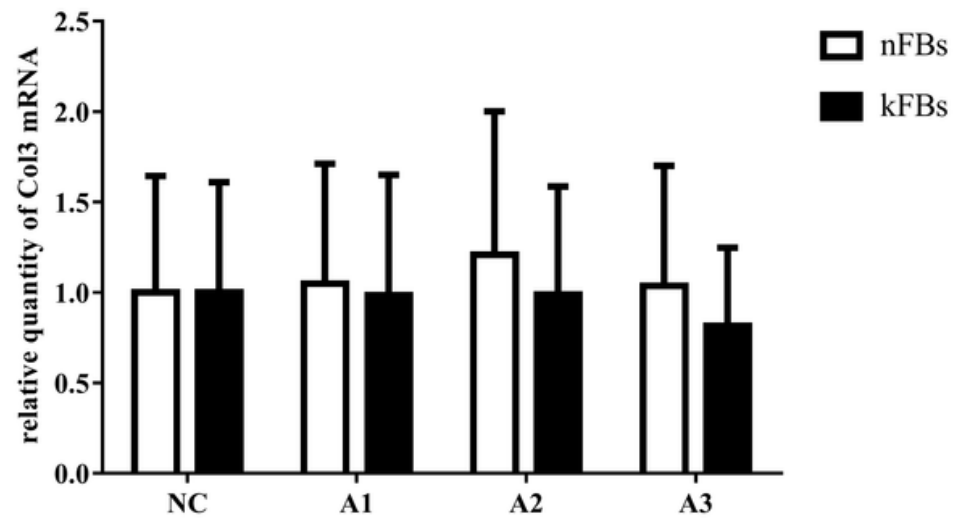

P-4-HB

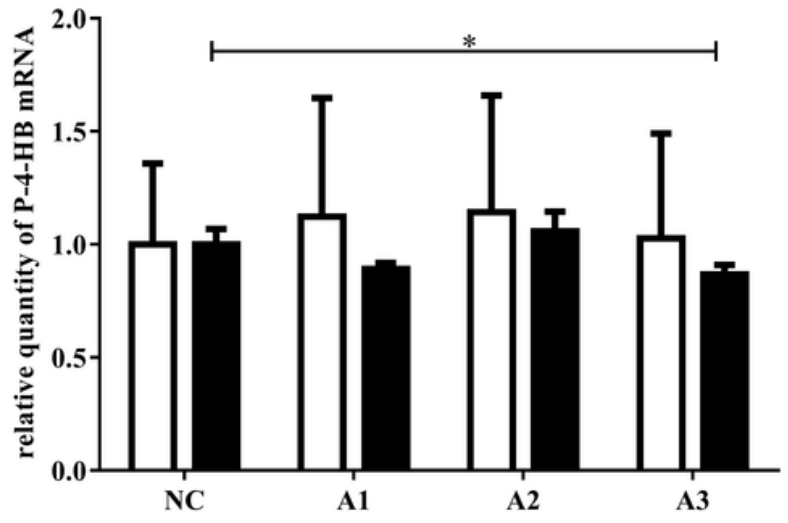

Figure 5

mRNA expressions of Col-1, Col-3, CTGF, and P-4-HB. The mRNA expression of Col-1 in kFBs was downregulated in co-cultural groups with $\mathrm{A} 2$ compared to nFBs. When co-cultured with $\mathrm{A} 3$, the P-4-HB expression was suppressed in kFBs, compared to kFBs in the NC group. ${ }^{*} p<0.05$. A: adipose-derived stem cells. nFBs: normal fibroblasts; kFBs: keloid fibroblasts. NC: normal control. Col: collagen. CTGF: connective tissue growth factor. P-4-HB: Prolyl 4-hydroxylase B. 


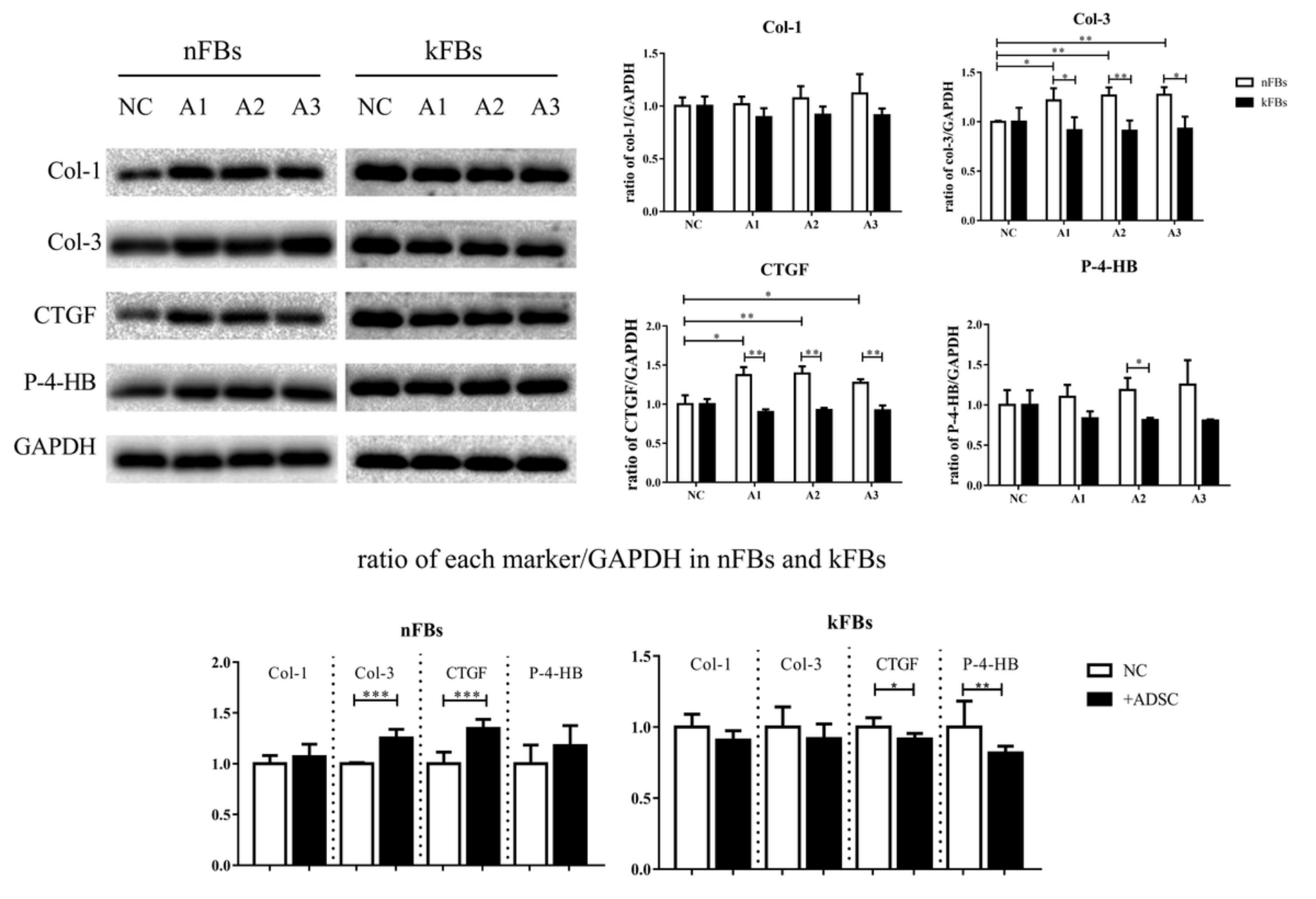

\section{Figure 6}

Protein expressions of Col-1, Col-3, CTGF, and P-4-HB. The protein levels of Col-3, CTGF, and P-4-HB were lower in kFBs co-cultured with ADSC than in $\mathrm{nFBs}$ under the same condition. The Col-1 protein level was not altered in co-cultured groups. The Col-3 and CTGF protein level of nFBs co-cultured with ADSC were higher than $\mathrm{nFBs}$ in NC groups. The CTGF and P-4-HB protein level of kFBs co-cultured with ADSC were lower than kFBs in NC groups. ${ }^{*} p<0.05 ;{ }^{* \star} p<0.005$; ${ }^{* \star} \mathrm{p}<0.001$. A, ADSC: human adipose-derived stem cells. nFBs: normal fibroblasts; kFBs: keloid fibroblasts. NC: normal control. Col: collagen. CTGF: connective tissue growth factor. P-4-HB: Prolyl 4-hydroxylase B. 


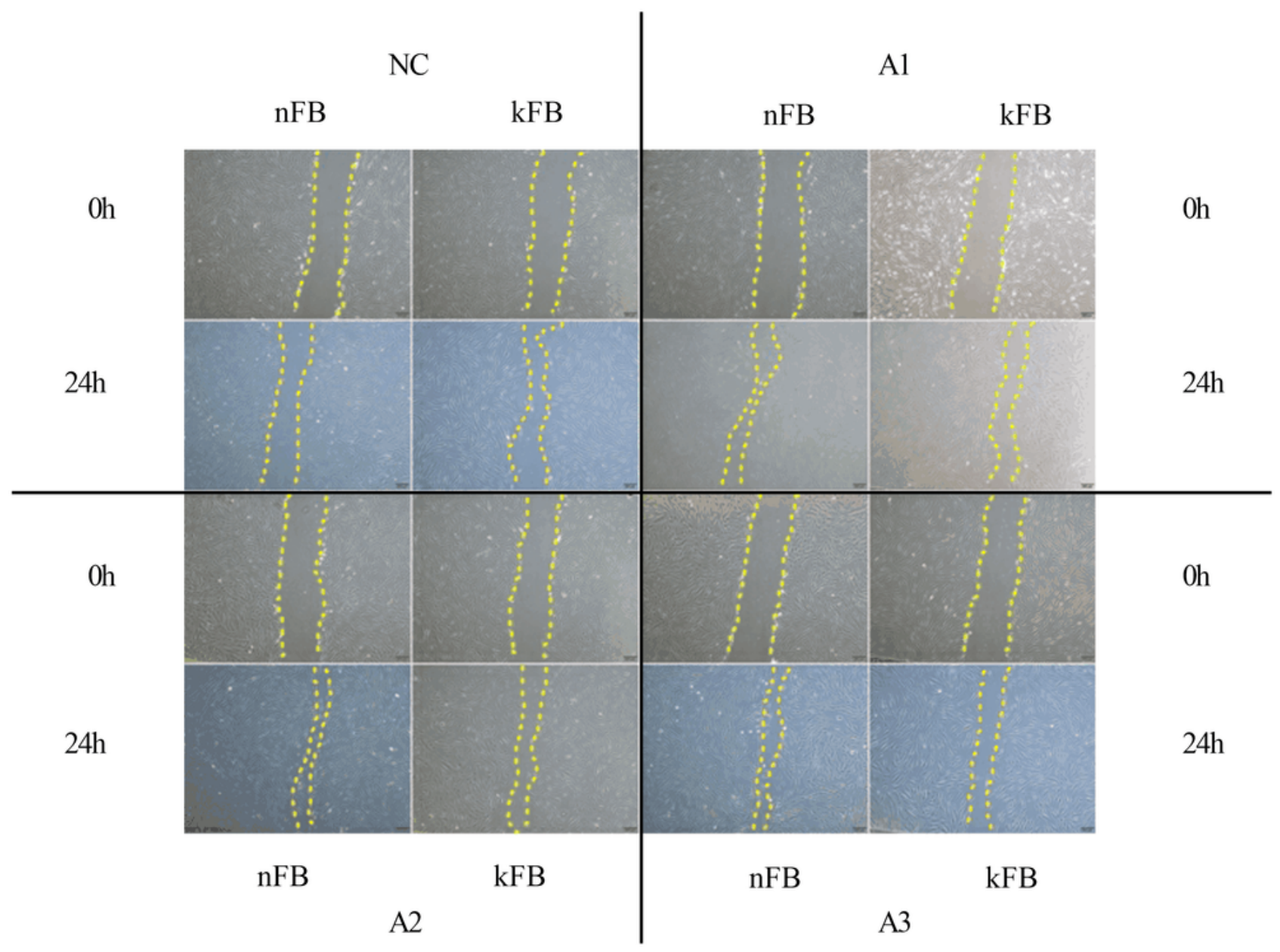

closure rates of scratches $(\%)$
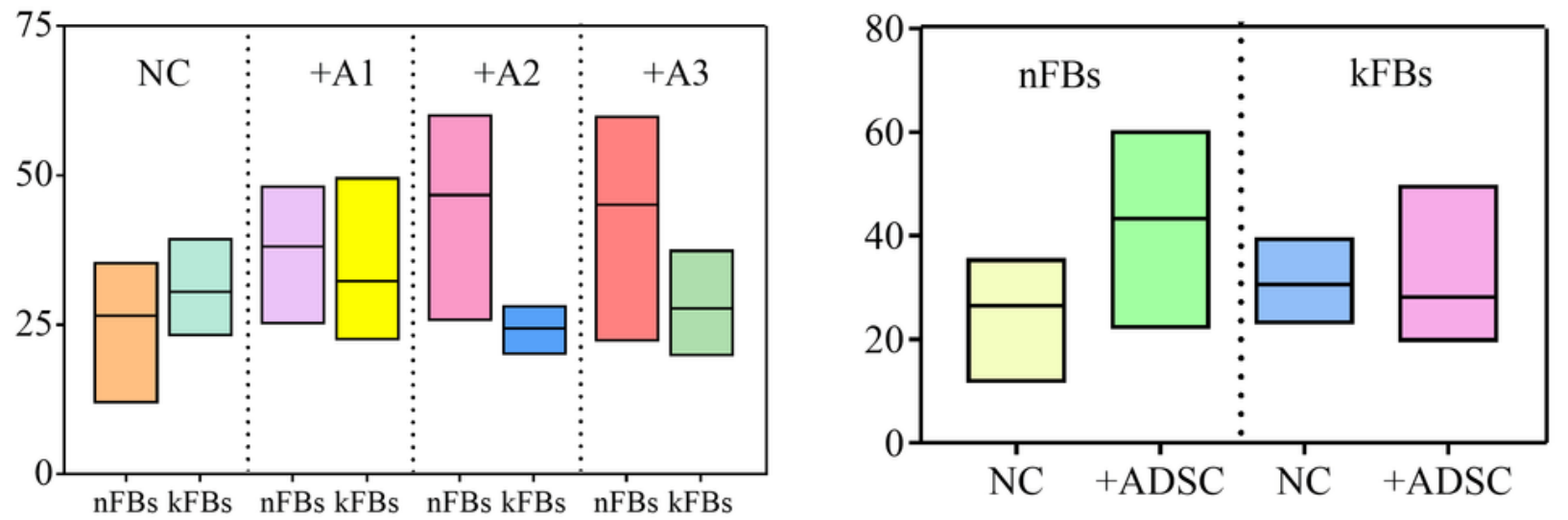

\section{Figure 7}

The closure of scratches. The scratches in $\mathrm{nFBs}$ and $\mathrm{kFBs}$ were all closed via cellular migration and proliferation after 24-hour culture in the NC group and co-cultured groups. However, no significant differences in closure rate were detected. FB: fibroblast. NC: normal control. A, ADSC: human adiposederived stem cells. nFBs: normal fibroblasts; kFBs: keloid fibroblasts. NC: normal control. 


\section{Supplementary Files}

This is a list of supplementary files associated with this preprint. Click to download.

- SuppleFig1.tif

- SuppleFig2.tif

- SuppleFig3.tif 